\title{
THE
}

\section{Using Golden Ratio Search to Improve Paired Construction of Quality Control Charts}

Xia Pan

Jeffrey E. Jarrett

University of Rhode Island, jejarrett133@outlook.com

Follow this and additional works at: https://digitalcommons.uri.edu/cba_facpubs

\section{Citation/Publisher Attribution}

Pan, Xia and Jarrett, Jeffrey. "Using Golden Ratio Search to Improve Paired Construction of Quality Control Charts." International Journal of Economics and Management Engeineering 3.3 (2013): 107-11. Available at http://www.ijeme.org/paperInfo.aspx?PaperlD=5226.

This Article is brought to you for free and open access by the College of Business at DigitalCommons@URI. It has been accepted for inclusion in College of Business Faculty Publications by an authorized administrator of DigitalCommons@URI. For more information, please contact digitalcommons-group@uri.edu. 


\title{
Using Golden Ratio Search to Improve Paired Construction of Quality Control Charts
}

\author{
Xia $\operatorname{Pan}^{1}$, Jeffrey E. Jarrett ${ }^{* 2}$ \\ ${ }^{1}$ Lingnan College, Sun Yat-sen University, Guangzhou, China \\ ${ }^{* 2}$ Management Science, CBA, University of Rhode Island, Kingston, RI 02881, USA \\ 1'panpapers@yahoo.com; ${ }^{2}$ jejarret@mail.uri.edu
}

\begin{abstract}
Our purpose is to indicate a new method for determining the control limits of univariate control charts to show the effectiveness of golden ratios search. We examine a solution to a problem when signals from mean and variance charts differ. Lack of concordance in the signals from mean and variance (or standard deviation) control charts bring confusion to Quality Control managers which in turn may lead to sub optimal management quality practices. To achieve better quality management practice, we provide a solution to the problem of finding different decision signals for in-control processes for quality control charts for mean and variability. We construct the control charts in experimental conditions for in-control average run length using the methods of simulation. Finally, we employ the golden ratio search method to identify the control limit parameters which differ from standard methods for constructing quality control charts. Last, we minimize the length of time in computation in the construction of these new quality control charts.
\end{abstract}

Keywords- Golden Ratio Search; Monte Carlo Method; SQC; Range and Standard Deviation Control Charts

\section{INTRODUCTION AND PURPOSE}

In SQC, a key measure for control chart performance is average run length (ARL). Its computation is usually determined by (1) Monte Carlo simulation, (2) Markov Chains, or (3) the calculus of integral equations. Due to the complexity of Markov Chains and the calculus of integral equations, we prefer the simpler Monte Carlo method. Also, due to advances in computer technology, simulation became the primary approach to estimate ARL (Woodall and Montgomery, 1999). However, some SQC simulation studies often require extensive computing time and frequent trials. In these cases, efficiency in the Monte Carlo approach is still a vital issue. Hence, our goal is to improve the efficiency of simulation and this goal is consistent with others, Berman (1997/1998), Nelson and Goldsman (2001), Fu (2002), Boesel et al (2003) and Gerdes et al (2005). In another study Pan, Jarrett and Mangiamelli (2001) explored the use of the geometric distribution to calculate the mean and standard deviation of run length. Although promising, this attempt did not explain why the average runs length (ARL) changes.

The importance of finding a solution to problems of conflicting signals will benefit quality management officers in a manner not heretofore examined deeply in quality management literature. We, further determine a solution when managers feel it necessary to treat the issue of concordance. Our solution defines an objective function as the absolute difference between the ARL of the variability control chart and the corresponding arithmetic mean chart. By applying the golden ration search we approximate the solution. The objective function is uni-modal with respect to lambda. Last formulate an easy to solve minimization problem and applies a well-known minimization method. Last, we find that the mathematical difficulties are not easily solved, but assist in serving quality managers with a solution that diminishes the concordance problem associated with differing signals from mean and variability control charts.

We suggest a method that is already known, but not previously employed to optimize the efficiency of simulation in SQC applications. By applying the golden ratio search (see Dunlap, 1997, van Zanten, 1999, and Weisstein, 2006) to reduce the number of trials, we produce a better method to approach the construction of SQC charts to control and improve the quality of manufactured output. Minimizing the total length of simulation time in the Monte Carlo approach will provide a better table of factors for constructing control limits for Shewhart type quality control charts. In turn, we recommend their inclusion in quality control handbooks for practical use to replace the conventional factors used for many decades.

\section{THE ISSUE OF CONCORDANCE}

Standard construction methods for SQC Charts, by, Montgomery (2005), Gaither and Frazier, (2002), and Duncan, (1986), among others, use factor tables to produce range (R) and standard deviation (S) control charts. The factors as $\mathrm{D}_{3}$ and $\mathrm{D}_{4}$ for $R$ charts, and $\mathrm{B}_{3}$ and $\mathrm{B}_{4}$ for $S$ charts are derived for known processes. However, in reality, most process parameters are unknown and we need to estimate them instead. The results are different for known and unknown processes. However, researchers sight to often the issue of concordance. That is, the mean and variability charts should lead to quality decisions that agree with each other by providing the same false signal rate when the process is in-control.

In standard Shewhart control charts, both the control charts for mean ( $\bar{X}$ charts) and for variability (Range, $R$, and Standard Deviation, $S$ ) usually set control limits at plus and minus "three-sigma" where the probability of a false signal $\alpha=$ 0.0027 , and corresponding average run length ARL is 370 , which is simply $1 / 0.0027$. If the underlying process has known 
parameters, we measure chart performance with the probabilities of false signals. When one estimates the process parameters from sample data, the probability of a false signal will be different from 0.0027 and the ARL is not simply related to the probability of a false signal. In this case, we should construct the control charts based ARL. Under the conventional settings, mean and corresponding variability charts do not have the same ARL for in-control processes. The issue of concordance arises because we desire that the ARL agree. This requires adjustment of control limits. In this paper, we adjust control limits for variability and leave the mean chart alone. We discuss only the estimated parameter cases since they are practical and useful.

The construction of quality control charts includes two phases. In Phase I, we collect samples from an in-control process to estimate the parameters for mean and variability. Phase II, we monitor the process with the constructed control charts. For variability charts conventionally set at three-sigma for an estimated process, the control limits are $U \hat{C} L_{\bar{R}}=\bar{R}+3 \hat{\sigma}_{R}=D_{3} \bar{R}$ and $L \hat{C} L_{\bar{R}}=\bar{R}-3 \hat{\sigma}_{R}=D_{4} \bar{R}$ with central line at $\bar{R}$, and $U \hat{C} L_{\bar{S}}=\bar{S}+3 \hat{\sigma}_{S}=B \bar{S}$ and $L \hat{C} L_{\bar{S}}=\bar{S}-3 \hat{\sigma}_{S}=B_{4} \bar{S}$ with central line at $\bar{S}$. These variability charts have different ARL from $\bar{X}$ charts. To adjust control limits, we use the number of (estimated) sigma $\lambda$, so that $U \hat{C} L_{\bar{R}}=\bar{R}+\lambda \hat{\sigma}_{R}=D_{3}^{\prime} \bar{R}$ and $L \hat{C} L_{\bar{R}}=\bar{R}-3 \hat{\sigma}_{R}=D_{4}^{\prime} \bar{R}$ with central line at $\bar{R}$, and $U \hat{C} L_{\bar{S}}=\bar{S}+\lambda \hat{\sigma}_{S}=B_{3}^{\prime} \bar{S}, L \hat{C} L_{\bar{S}}=\bar{S}-\lambda \hat{\sigma}_{S}=B_{4}^{\prime} \bar{S}$. Through Monte Carlo simulations for different Phase I sampling plans, we obtain the appropriate $\lambda$ for $R$ charts and $S$ charts so that $A R L$ 's will concord for both the mean and variability charts.

To adjust properly, we require a value for $\lambda$ that may lead to different factor values of $B_{3}, B_{4}, D_{3} D_{4}$ that are given in conventional textbooks and handbooks. The goal of an adjustment is to make certain the absolute difference between the adjusted ARL's of the mean and variability control charts are sufficiently small. This usually requires many trials before we reach a desired optimum $(\lambda)$. Thus, we have a unimodal optimization problem searching for the optimal value of $\lambda$.

A unimodal optimization is a process that searches the optimal point of a meaningful (continuous) object function where only one optimal point exists in a given range. Assuming the underlying process is normal and there is no evidence of serial correlation in a process control illustration, for each pair of Phase I number of samples $m$ and sample size $n$, there is one such unimodal search problem. Our simulations are run for Phase I plan when $\mathrm{m}=20,30$, 50, and 100, and $\mathrm{n}=3,4 \ldots 8$, and 10 . Modern personal computers will run several hours, if not days, for each pair of $(m, n)$. As we have so many pairs of (m, $n$ ) and for each pair the number of times of adjustment of $\lambda$ is probably uncertain and very time consuming. We need an efficient search method and we propose using golden ratio search to achieve this goal.

\section{GOLDEN RATIO SEARCH}

Golden ratio search is effective in unimodal optimization because it results in the least number of searches or trials to locate the optimum. We find mathematical studies of golden ratio search in Wilde and Beightler (1987), Livio (2002), among others. Given a unimodal object function defined in a starting range $\left[a_{0}, b_{0}\right]$, to search step-by-step, one condenses the range in which the optimal point is located until the width of the range is less than the given accuracy to position the location. Golden ratio search is the use of the golden section ratio 0.618 , or symmetrically, $(1-0.618)=0.382$, to condense the width of the range in each step.

Specifically, at each step of the search, the next sampling point is the 0.618 section point of the range (width) of the current step. For example, if the current range is $\left[a_{\mathrm{i}}, b_{\mathrm{i}}\right]$, the width of the next step becomes $0.618\left(b_{\mathrm{i}}-a_{\mathrm{i}}\right)$. One of the end-points of this new range is either $a_{\mathrm{i}}$ or $b_{\mathrm{i}}$, depending on the magnitudes of the object function at $a_{\mathrm{i}}$ and $b_{\mathrm{i}}$. The other end-point of the new range is the new checking point, located by the golden ratio 0.618 . The search stops when the width of the current range is less than the preset accuracy. If we define accuracy as the percentage of the width of the starting range $\left[a_{0}, b_{0}\right]$, golden ratio search always requires the same number of checking points for certain accuracy, no matter where the maximum point is actually located--as long as it is within $\left(a_{0}, b_{0}\right)$. Once a target range's width is less than or equal to accuracy, the search ends. For example, if the accuracy is $1 \%$ of the width of $\left[a_{0}, b_{0}\right]$, eleven such search observations were necessary. If the accuracy is $10 \%$ of the original width, we needed only seven search observations. Given the accuracy, the number of observations is determined regardless of where we locate the optimum in $\left[a_{0}, b_{0}\right]$ and the shape of the objective function.

Initially, we obtain one of the two edge points of the starting range from the ARL comparison of mean and variability charts. The difference in ARL also indicates the direction of the adjustment in control limits for variability charts. To obtain the original (starting) range we need one or two trial adjustments to yield the other edge point of the starting range. For example, we begin the settings with plus and minus three-sigma limits for the two control charts, i.e. six-sigma. If the ARL of an $R$ (or $\mathrm{S}$ ) chart is smaller than the ARL of its corresponding $\bar{X}$ chart, then the value of $\lambda$ is adjusted larger. Based on some prior experience, $\lambda=3.3$ is usually aggressive enough to assess the other edge point of the starting range. As long as the ARL of $R$ chart at $\lambda=3.3$ is larger than the ARL of the $\bar{X}$ chart, we obtain the other edge point of the starting range. Thus, the starting range for the search is $\left[\lambda_{\min }, \lambda_{\max }\right]=[3,3.3]$. Note the key here is not the magnitude of the width at the starting range, but to 
obtain a range for starting purposes. Golden ratio search will quickly reduce the target range even if the starting range is very wide.

When we utilize golden ratio search, the objective function is the absolute difference between the ARL of a variability chart and the corresponding $\bar{X}$ chart. This objective function is unimodal with respect to $\lambda$ and has a minimum of zero when the two ARL's are equal. We can designate this function as F ( $\lambda$ ). The ARL of the $\bar{X}$ chart, denoted as ARL0, is a certain target value for a given Phase I sampling $(\mathrm{m}, \mathrm{n})$. We require the starting range ARL0 to be within [ARL $\left(\lambda_{\min }\right)$, ARL $\left.\left(\lambda_{\max }\right)\right]$. The objective function is defined as $F(\lambda)=\mid \operatorname{ARL}(\lambda)$ - ARL0 $\mid$. Since ARL $(\lambda)$ is monotonically increasing with respect to $\lambda$, then $F(\lambda)$ must be unimodal if ARL0 is a value within $\left[\operatorname{ARL}\left(\lambda_{\min }\right)\right.$, ARL $\left.\left(\lambda_{\max }\right)\right]$.

For example, with the starting range [3, 3.3], the next check points are at $3+(3.3-3) * 0.618=3.1854$ and $3.3-(3.3-$ $3)^{*} 0.618=3.1146$. Comparing the magnitude of the object function at these four points, there are two possible situations. Situation A, if $F(3)>F(3.1146)>F(3.1854)$, then we discard the point at $\lambda=3$, and for the next step, the range is [3.1146, 3.3]. Situation B, if $F(3.1146)<F(3.1854)<F(3.3)$, then we discard the point at $\lambda=3.3$, and for the next step the range is [3, 3.1854]. Either Situation A or B occurs because the F $(\lambda)$ is unimodal in [3, 3.3]. Based on the new range, repeating the procedure narrows the range in the following sequence. The search stops once the new range is narrower than the preset accuracy width.

As ARL of a chart is monotonic of $\lambda$, there is unique correspondence between the ARL and $\lambda$; hence the accuracy width for search can be set at either a width of $\lambda$ or a width of ARL. Since $F(\lambda)=\mid$ ARL $(\lambda)-$ ARL0 $\mid$ is nonnegative with the minimum of zero, setting accuracy as the width of ARL is equivalent to setting a maximum allowed value of $F(\lambda)$ to stop the search. In this study, the golden ratio search stops when F $(\lambda)$ is less than the preset threshold. The preset threshold is set as the sum of the standard error of ARL0 and the standard error of ARL $(\lambda)$, while these standard errors as a result of simulation are controlled to be no more than 6 . There is no special reason to choose this value of 6 . You can choose any other desired standard error by changing the number of repetitions in simulation. Given the accuracy set above, golden ratio search locates the target ARL of variability charts with the minimum and certain number of adjustment trials.

\section{RESULTS OF EXPERIMENTS}

Table 1 shows the computed ARL's of mean charts by Monte Carlo simulation. [For a complete discussion of ARL and its measure see Montgomery, (2005), pp. 160,218,220,236,287-288, 386, 395-398, 411-412, and 435.] We show in Table 1 (a) the ARL's of $\bar{X}$ charts with estimated control limits estimated using the mean range $(\bar{R})$. Table 1(b) contains the same limits based on the estimated standard deviation $(\bar{S}$ ). It is well known that false alarm rates differ in X-bar charts based on estimated R and S. Note the relative closeness of the values for the ARL in Table 1 Parts (a) and (b) when comparing the each pair of $\boldsymbol{m}$ (number of samples) and $\boldsymbol{n}$ (samples size). These ARLs of the mean charts are quite different from 370 , which is the property of the charts for known process. For the same reason, we are convinced that there is no way the ARLs of the variability charts (the values not reported here) are 370, and they must also be different from the ARLs in Table 1.

TABLE I (A) 95\% CONFIDENCE INTERVAL OF ARL'S FOR $\bar{X}$ CHARTS WITH 3 SIGMA SETTING, ESTIMATED WITH $\bar{R}$

\begin{tabular}{ccccc}
\hline & \multicolumn{5}{c}{$\mathrm{m}$} \\
\cline { 2 - 5 } & 20 & 30 & 50 & 100 \\
\hline 3 & $(710,730)$ & $(538,558)$ & $(447,467)$ & $(400,420)$ \\
4 & $(510,530)$ & $(445,465)$ & $(403,423)$ & $(378,398)$ \\
5 & $(455,475)$ & $(410,430)$ & $(378,398)$ & $(375,395)$ \\
\hline
\end{tabular}

( $\mathrm{m}$ is the number of samples in Phase $\mathrm{I}, \mathrm{n}$ is sample size.)

TABLE I (B) 95\% CONFIDENCE INTERVAL OF ARL'S FOR $\bar{X}$ CHARTS WITH 3 SIGMA SETTING, ESTIMATED WITH $\bar{S}$

\begin{tabular}{ccccc}
\hline $\mathrm{n}$ & 20 & 30 & 50 & 100 \\
\cline { 2 - 5 } & $(710,730)$ & $(538,558)$ & $(442,462)$ & $(405,425)$ \\
3 & $(505,525)$ & $(440,460)$ & $(405,425)$ & $(378,398)$ \\
4 & $(440,460)$ & $(398,418)$ & $(376,396)$ & $(372,392)$ \\
\hline
\end{tabular}

( $\mathrm{m}$ is the number of samples in Phase $\mathrm{I}, \mathrm{n}$ is sample size.)

Therefore, if we follow conventional construction of control charts, the ARL of the chart for the detection of changes in mean and the ARL of the corresponding chart for the detection of changes in variability are not in agreement, when the process is actually in control.

Table 2 contains the remedy to the problem. One adjusts the control limits of the variability charts to have the same ARL as the corresponding mean charts. We made the adjustments with the golden ratio search method described in the above section 
of this paper. Table 2 (a) is the adjustment of $R$ charts and Table 2 (b) is the adjustment of $S$ charts. We report the 95\% confidence interval of the suggested construction factors of $B_{3}^{\prime}, B_{4}^{\prime}, D_{3}^{\prime} D_{4}^{\prime}$ to replace the conventional values of $B_{3}, B_{4}, D_{3} D_{4}$ given in textbooks and handbooks. As noted previously, the simulations using golden ratio search to find the target adjustment as the difference in ARL for the pair of mean and adjusted variability charts is less than the sum of the ARL standard errors of the pair.

TABLE II (A) R CHARTS ADJUSTED IN CONCORDANCE TO $\bar{X}$ CONTROL CHARTS

\begin{tabular}{|c|c|c|c|c|c|}
\hline \multirow{3}{*}{$\mathrm{n}$} & Factor/ & \multicolumn{5}{|c|}{$\mathrm{m}$} \\
\cline { 3 - 6 } & Parameter & 20 & 30 & 50 & 100 \\
\hline \multirow{5}{*}{3} & ARL & $(708,728)$ & $(530,550)$ & $(434,454)$ & $(400,420)$ \\
& $D_{3}^{\prime}$ & 0 & 0 & 0 & 0 \\
& $\left(D_{3}\right)$ & $(0)$ & $(0)$ & $(0)$ & $(0)$ \\
\cline { 3 - 6 } & $D_{4}^{\prime}$ & 2.658 & 2.689 & 2.710 & 2.741 \\
& $\left(D_{4}\right)$ & $(2.575)$ & $(2.575)$ & $(2.575)$ & $(2.575)$ \\
\hline \multirow{5}{*}{4} & ARL & $(510,530)$ & $(443,463)$ & $(408,428)$ & $(378,398)$ \\
\cline { 3 - 6 } & $D_{3}^{\prime}$ & 0 & 0 & 0 & 0 \\
& $\left(D_{3}\right)$ & $(0)$ & $(0)$ & $(0)$ & $(0)$ \\
& $D_{4}^{\prime}$ & 2.308 & 2.338 & 2.368 & 2.376 \\
& $\left(D_{4}\right)$ & $(2.282)$ & $(2.282)$ & $(2.282)$ & $(2.282)$ \\
\hline \multirow{5}{*}{5} & ARL & $(450,470)$ & $(405,425)$ & $(371,391)$ & $(370,390)$ \\
\cline { 3 - 6 } & $D_{3}^{\prime}$ & 0 & 0 & 0 & 0 \\
& $\left(D_{3}\right)$ & $(0)$ & $(0)$ & $(0)$ & $(0)$ \\
& $D_{4}^{\prime}$ & 2.126 & 2.148 & 2.159 & 2.189 \\
& $\left(D_{4}\right)$ & $(2.115)$ & $(2.115)$ & $(2.115)$ & $(2.115)$ \\
\hline
\end{tabular}

( $\mathrm{m}$ is the number of samples in Phase $\mathrm{I}, \mathrm{n}$ is sample size. The factors give ARLs at $95 \%$ confidence interval. Values for $D_{4}$ in parentheses are unadjusted.)

TABLE II (B) S CHARTS ADJUSTED IN CONCORDANCE TO $\bar{X}$ CHARTS

\begin{tabular}{|c|c|c|c|c|c|}
\hline \multirow{2}{*}{$\mathrm{n}$} & \multirow{2}{*}{$\begin{array}{c}\text { Factor/ } \\
\text { Parameter }\end{array}$} & \multicolumn{4}{|c|}{$m$} \\
\hline & & 20 & 30 & 50 & 100 \\
\hline \multirow{3}{*}{3} & ARL & $(700,720)$ & $(543,563)$ & $(445,465)$ & $(412,432)$ \\
\hline & $\begin{array}{l}B_{3}^{\prime} \\
\left(B_{3}\right)\end{array}$ & $\begin{array}{c}0 \\
(0)\end{array}$ & $\begin{array}{c}0 \\
(0)\end{array}$ & $\begin{array}{c}0 \\
(0)\end{array}$ & $\begin{array}{c}0 \\
(0)\end{array}$ \\
\hline & $\begin{array}{l}B_{4}^{\prime} \\
\left(B_{4}\right)\end{array}$ & $\begin{array}{c}2.631 \\
(2.568)\end{array}$ & $\begin{array}{c}2.668 \\
(2.568)\end{array}$ & $\begin{array}{c}2.694 \\
(2.568)\end{array}$ & $\begin{array}{c}2.725 \\
(2.568)\end{array}$ \\
\hline \multirow{3}{*}{4} & ARL & $(514,534)$ & $(442,462)$ & $(403,423)$ & $(365,385)$ \\
\hline & $\begin{array}{l}B_{3}^{\prime} \\
\left(B_{3}\right)\end{array}$ & $\begin{array}{c}0 \\
(0)\end{array}$ & $\begin{array}{c}0 \\
(0)\end{array}$ & $\begin{array}{c}0 \\
(0)\end{array}$ & $\begin{array}{c}0 \\
(0)\end{array}$ \\
\hline & $\begin{array}{l}B_{4}^{\prime} \\
\left(B_{4}\right)\end{array}$ & $\begin{array}{c}2.262 \\
(2.266)\end{array}$ & $\begin{array}{c}2.287 \\
(2.266)\end{array}$ & $\begin{array}{c}2.317 \\
(2.266)\end{array}$ & $\begin{array}{c}2.334 \\
(2.266)\end{array}$ \\
\hline \multirow{3}{*}{5} & ARL & $(440,460)$ & $(400,420)$ & $(375,395)$ & $(372,382)$ \\
\hline & $\begin{array}{l}B_{3}^{\prime} \\
\left(B_{3}\right)\end{array}$ & $\begin{array}{c}0 \\
(0)\end{array}$ & $\begin{array}{c}0 \\
(0)\end{array}$ & $\begin{array}{c}0 \\
(0)\end{array}$ & $\begin{array}{c}0 \\
(0)\end{array}$ \\
\hline & $\begin{array}{l}B_{4}^{\prime} \\
\left(B_{4}\right)\end{array}$ & $\begin{array}{c}2.064 \\
(2.089)\end{array}$ & $\begin{array}{c}2.089 \\
(2.089)\end{array}$ & $\begin{array}{c}2.107 \\
(2.089)\end{array}$ & $\begin{array}{c}2.125 \\
(2.089)\end{array}$ \\
\hline
\end{tabular}

( $m$ is the number of samples in Phase $\mathrm{I}, \mathrm{n}$ is sample size. The factors give ARLs at $95 \%$ confidence interval. Values for $B_{4}$ in parentheses are unadjusted.)

\section{SUMMARY AND DISCUSSION}

We show the use of golden ratio search in simulation studies associated with SQC. With golden ratio search, we minimize the computing time to find an optimal solution. Without golden ratio search, the time needed to find an optimal solution is greater and very costly. The study allowed us to efficiently obtain a new table, Table 2, containing factors for constructing 
variability control charts that result in signal agreement with control charts for mean. We recommend this new table as an addition to handbooks on SQC and related disciplines for practical use.

Further, this golden ratio methodology is better than explaining and calculating control limits noted before in Pan, Jarrett, and Mangiamelli (2001). The methodology at that time produced results that (1) the run length behavior contained greater skewness if control limits were estimated; (2) decisions as the use of $\mathrm{S}$ and $\mathrm{R}$ charts were not fully developed; and (3) adjustment coefficients for $\mathrm{R}$ charts were not precise which could lead to less than useful results.

One can apply golden ratio search in simulation to other applications in the event of a unimodal objective function. If simulation has a goal of efficiency, we construct the absolute difference between the simulated value and the goal value as the objective function for golden ratio search. Although increases in the speed and power of computer technology tend to occur, studies like this still suggest golden ratio search fulfills the objectives of efficiency and better results. In the future, we expect more economies of scale in producing efficient and concordant quality control procedures using mathematical application such as the golden ratio search.

\section{REFERENCES}

[1] Berman, L. (1997/1998) “Accelerating Monte Carlo: quasirandom sequences and variance reduction,” Computational Finance 1(2) winter.

[2] Boesel, J., B.L. Nelson, N. Ishii (2003) “A framework for simulation-optimization software,” IIE Transactions 36(3) 221-229.

[3] Champ, C.W. (1992) "Steady-state run length analysis of a Shewhart quality control chart with supplementary runs rules," Communications in Statistics- Theory and Methods 21(3) 765-777.

[4] Deming, W. E. (1986). Out of Crisis, MIT Press, Cambridge, MA.

[5] Divorky, J.J. and Taylor, R.W. (1995) Detecting process drift with combinations of trend and zonal supplementary runs rules," International Journal of Quality and Reliability Management 12, 2, 60-71.

[6] Duncan, A. J. (1986). Quality Control and Industrial Statistics, 5th ed., Irwin Inc., IL.

[7] Dunlap, R.A. (1997) The golden ratio and Fibonacci numbers. World Scientific Publishing Co., Inc., River Edge, NJ, (USA) 162.

[8] Fu, M.C. (2002) “Optimization for simulation: Theory vs. practice,” INFORMS Journal of Computation 13(3) 192-215.

[9] Gaither, N. and Frazier, G. (2002). Operations Management, 9th ed., Southwestern, Cincinnati, OH (USA).

[10] Gerdes, M., T. Barth, and M. Grauer (2005) "Performance analysis of distributed solution approaches to simulation-based optimization," Computational Management Science 2(1), 57-82.

[11] Livio, M. The Golden Ratio: The Story of Phi, the World's Most Astonishing Number, New York: Broadway Books.

[12] Montgomery, D. C. (2005) Introduction to Statistical Quality Control, 5th ed., John Wiley \& Sons Inc., New York.

[13] Nelson, B.L., and D.Goldsman (2001) “Comparisons with a standard in simulation experiments," Management Science 47, $449-463$.

[14] Pan, X. Jarrett, J.E. and Mangiamelli, P. (2001) "The Adjustment for Average Run Length Consistency between Mean Chart and Variability Chart," 2001 Proceedings of the Decision Sciences Institute, Atlanta, GA (USA).

[15] Shewhart, W. A. (1931). Economic Control of Quality of Manufactured Product, D. Van

[16] Nostrand Company, Inc., New York.

[17] Van Zanten, A. J. (1999) "The Golden Ratio in the Arts of Painting, Building, and Mathematics.” Nieuw Arch. Wisk. 17, $229-245$.

[18] Weisstein, E W. (2006) "Golden Ratio" From MathWorld--A Wolfram Web Resource, http://mathworld.wolfram.com/GoldenRatio.html.

[19] Wilde, D. J. and Beightler, B.S. (1967) Foundations of Optimization. Prentice-Hall Inc. New Jersey.

[20] Woodall, W. H. and Montgomery, D. C. (1999). "Research Issues and Ideas in Statistical Process Control", Journal of Quality Technology. 31, 4,376-386.

[21] Zhang, S. and Wu, Z. (2005) "Designs of control charts with supplementary runs rules" Computers \& Industrial Engineering 49(1) 76-97. 